\title{
Recuperação Desenvolvimental Após a Adoção: Características da Criança e da Família Adotiva
}

\author{
Developmental Recovery After Adoption: Child and Adoptive \\ Family's Characteristics
}

\author{
Joana Baptista*, ${ }^{*}$, Isabel Soares ${ }^{b} \&$ Margarida Henriques $^{a}$ \\ ${ }^{a}$ Faculdade de Psicologia e de Ciências da Educação da Universidade do Porto, Porto, Portugal \\ $\&^{b}$ Escola de Psicologia, Universidade do Minho, Braga, Portugal
}

\begin{abstract}
Resumo
Clínicos e investigadores têm alertado para os efeitos problemáticos das experiências precoces adversas, nomeadamente a da institucionalização, no crescimento físico, no desenvolvimento cognitivo e sócio-emocional, bem como nas relações de apego da criança. Após a adoção, a recuperação nestes domínios parece ser evidente, observando-se uma curiosa heterogeneidade nos resultados desenvolvimentais. Este artigo de revisão apresenta uma sistematização de resultados de estudos que analisaram a influência das características da criança e da família adotiva naquela recuperação. Pesquisou-se nas principais bases de dados eletrônicas e foram identificados estudos originais, de revisão e capítulos de livros. Concluiu-se que um entendimento da recuperação exige a exploração dos fatores genéticos, das experiências pré-natais e pré-institucionalização, e das vivências de cuidados anteriores e posteriores à adoção.

Palavras-chave: Desenvolvimento, adoção, criança, família.

Abstract

Clinicians and researchers have been warning about the harmful effects of a history marked by early adverse experiences in shelter institutions in child's health, cognitive and socioemotional development as well as attachment. After adoption, the recovery in these domains seems to be clear and it is also observed a curious heterogeneity in developmental results. This paper review presents a summary of the main empirical findings about the characteristics of the child and of the adoptive family involved in the recovery process. The search was done in the major electronic databases and original studies, review articles, and book chapters were identified. It is concluded that the recovery requires the identification of genetic, prenatal and pre-institutionalization factors, and also the care experiences before and after the adoption.

Keywords: Development, adoption, child, family.
\end{abstract}

As crianças adotadas, em comparação com os pares da comunidade, experienciaram, nos primeiros tempos de vida, maior adversidade e risco ao nível das complicações ao nascimento e/ou ao nível das experiências de perda da família biológica (Rutter, 2005). Algumas foram ainda institucionalizadas em contextos marcados por cuidados não responsivos, com implicações nefastas para o desenvolvimento posterior, em áreas diversas, como o crescimento físico, o desenvolvimento neurobiológico, cognitivo e emocional (Van IJzendoorn \& Juffer, 2006). Diferentes estudos realizados em contexto institucional têm

\footnotetext{
${ }^{*}$ Endereço para correspondência: Faculdade de Psicologia e de Ciências da Educação, Universidade do Porto, Rua Alfredo Allen, Porto, Portugal 4200-135. E-mail: joana. baptista.mail@gmail.com, isoares@psi.uminho.pt e mrangel@fpce.up.pt

Este artigo foi redigido com o apoio da Fundação para a Ciência e Tecnologia - PTDC/PSI-PCL/101506/2008 e Fundação Bial - Bolsa 13/06
}

vindo a indicar que as instituições tendem a não apresentar as características de um contexto de cuidados capaz de proporcionar à criança a satisfação das suas necessidades básicas de proteção, de afeto e de exploração do ambiente. As instituições, devido (a) à reduzida capacidade de abarcar as necessidades individuais, (b) ao baixo número de cuidadores em proporção ao de crianças, e (c) às mudanças frequentes de turno, tendem a promover a escassez de oportunidades para a realização de atividades de promoção desenvolvimental e de interação entre a criança e um grupo de cuidadores estável (D. E. Johnson, 2000).

Estudos comparativos, desenhados para analisar as diferenças entre o contexto familiar e o institucional, relatam que as instituições frequentemente providenciam poucas oportunidades para a criança adquirir e praticar novas competências, sendo raras as ocasiões em que as práticas institucionais são adaptadas às necessidades individuais. Gunnar e Kertes (2005), entre outros (e.g., Zeanah, Smyke, Koga, Carlson, \& The Beip Core Group, 2005), consideram 
que mesmo em instituições que providenciam estimulação sensorial e social adequada, a ausência de oportunidades para a construção de uma relação consistente entre a criança e um adulto poderá ser também um obstáculo ao desenvolvimento futuro adaptado.

Após a adoção, parece haver persistência de dificuldades, em domínios como o desenvolvimento sócio-emocional e o desempenho escolar (Van IJzendoorn, Juffer, \& Klein-Poelhuis, 2005). Além disso, as experiências prévias em contexto institucional parecem contribuir para uma maior probabilidade de ocorrência de determinados problemas em crianças adotadas, tais como comportamentos atípicos (e.g., comportamentos estereotipados, características autísticas; Marcovitch, Cesaroni, Roberts, \& Swanson, 1995), comportamentos perturbados de apego (O'Connor \& Zeanah, 2003) e falta de atenção/sobreatividade (Rutter, Kreppner, O’Connor, \& The ERA Study Team, 2001). Os termos falta de atenção/sobreatividade foram introduzidos (Roy, Rutter, \& Pickles, 2000) para denotar a possibilidade de constituírem um problema diferente do que está descrito no DSM-IV-TR (SHDA; American Psychiatric Association, 2000). De acordo com Rutter et al. (2001), este problema estaria associado à experiência de privação vivida anteriormente em contexto institucional, designando-o como síndrome da pós-institucionalização. Autores têm vindo também a salientar o impacto de fatores genéticos e de experiências pré-institucionalização naqueles e noutros domínios desenvolvimento (e.g., Simmel, 2007; Wiik et al., 2011).

Contudo, a recuperação desenvolvimental após a adoção parece ser igualmente evidente em áreas como o crescimento físico (e.g., D. E. Johnson, 2000), o desenvolvimento cognitivo e da linguagem (e.g., Cohen, Lojkasek, Zadeh, Pugliese, \& Kiefer, 2008; Tan \& Yang, 2005) e a segurança do apego (Juffer \& Rosenboom, 1997). Estes resultados sugerem o efeito diferencial das experiências de privação conforme o domínio do desenvolvimento, bem como a presença de variações individuais no grau de recuperação posterior.

Os estudos sobre o impacto da adoção têm dado particular enfoque ao contributo exercido por duas variáveis na recuperação desenvolvimental: o tempo de institucionalização e o de permanência na família adotiva. Todavia, a investigação tem vindo também a analisar a influência de outras variáveis possivelmente envolvidas nos mecanismos explicativos da mudança ocorrida após a adoção e da variabilidade observada. Rutter (2005) salienta, para além das experiências pré-natais adversas e da qualidade dos cuidados prestados antes da adoção, a pertinência de outras variáveis, tais como (a) características atribuídas à criança (e.g., o estatuto desenvolvimental no momento da adoção, a suscetibilidade geneticamente programada à privação ambiental); e (b) a qualidade do ambiente familiar adotivo.

A privação precoce de cuidados parentais adequados é um tema social de relevância mundial. A este respeito, estima-se que cerca de oito milhões de crianças, prove- nientes de 93 países, estejam a viver em instituições (Joint United Nations Programme on HIV [UNAIDS], United Nations Children's Fund [UNICEF], \& United States Agency for International Development [USAID], 2004). Tendo por base uma perspectiva do desenvolvimento ecológico-sistêmica, que descreve o desenvolvimento humano como a acomodação progressiva e mútua, ao longo da vida, entre um organismo biopsicológico complexo e ativo e as propriedades em mudança dos contextos imediatos e distais que o envolvem (Bronfenbrenner, 1993), os autores colocaram as seguintes questões: quais as trajetórias de desenvolvimento destas crianças, precocemente privadas de cuidados adequados, quando integradas num contexto familiar? Quais serão as características individuais e as componentes deste contexto promotoras de mudança no desenvolvimento? Com vista a contribuir para o delineamento de futuras investigações, este artigo apresenta uma sistematização dos resultados de estudos que analisaram o impacto das características atribuídas à criança e à família adotiva nos domínios que têm sido objeto de interesse por parte da comunidade científica, no estudo da adoção: crescimento físico, desenvolvimento cognitivo e sócio-emocional e relações de apego da criança.

\section{Método}

A estratégia de busca de referências foi dividida em duas etapas. Durante a primeira etapa, pesquisou-se nas principais bases de dados eletrônicas, nomeadamente MEDLINE, PsycINFO e SciELO, entre os anos de 1945 a 2011. Foram utilizados como descritores os termos: adoção (adoption), adotado (adoptee), família adotiva (adoptive family), recuperação desenvolvimental após a adoção (developmental recovery after adoption), crianças pós-institucionalizadas (post-institutionalized children), crianças institucionalizadas (institution-reared children) e institucionalização (institutionalization). Além disso, foram analisadas as referências bibliográficas dos artigos. A segunda etapa foi dedicada à exclusão dos artigos não relevantes à pesquisa, através de uma análise do resumo e, em seguida, de uma avaliação mais detalhada dos mesmos. No que concerne aos critérios de inclusão, foram identificados estudos nacionais e internacionais, desde clássicos a atuais, que se debruçaram sobre a análise do impacto das características da criança e da família adotiva em domínios específicos, designadamente (a) no crescimento físico, (b) no desenvolvimento cognitivo e (c) sócio-emocional, e (d) nas relações de apego.

\section{Resultados}

Do total, foram encontradas 149 produções possivelmente relevantes e 46 foram selecionadas após uma avaliação detalhada (i.e., 40 estudos originais, três estudos de revisão e três capítulos de livros). Com base na proposta de Rutter (2005), os conteúdos de cada trabalho selecionado foram organizados em duas categorias. A primeira foi 
denominada de contributos da criança adotada, e engloba quer fatores genéticos e gênero da criança quer fatores correspondentes ao período que antecedeu a adoção (i.e., fatores pré-natais, experiências de maus-tratos e história de cuidados, tempo de institucionalização e idade na adoção, estatuto desenvolvimental prévio). A segunda categoria foi intitulada de contributos do contexto familiar adotivo e abarca as características dos pais adotivos exploradas nos trabalhos elegidos (i.e., sensibilidade parental e modelos internos de funcionamento).

\section{Contributos da Criança Adotada}

Fatores Genéticos. A constante falta de informação acerca do background familiar da criança adotada tem contribuído para que seja difícil examinar o impacto no desenvolvimento de determinadas variáveis, tais como a vulnerabilidade genética. No entanto, evidências recentes indicam que polimorfismos genéticos estão associados a dificuldades de atenção e hiperatividade após um período em acolhimento institucional. Stevens et al. (2009) testaram a hipótese de que a heterogeneidade encontrada na recuperação desenvolvimental após a adoção seria influenciada pela interação Genótipo X Ambiente (GXE) e que os polimorfismos genéticos envolvidos em um neurotransmissor, a dopamina, moderam os efeitos da privação institucional sobre aqueles problemas. Usando um desenho longitudinal, a hiperatividade e o déficit de atenção foram avaliados aos 6,11 e 15 anos, numa amostra de crianças adotadas por famílias do Reino Unido e que estiveram institucionalizadas até aos 42 meses de idade na Romênia. Os autores apuraram que o risco para evidenciar aquelas dificuldades, associado à institucionalização precoce, era moderado pelo gene que codifica o transportador de dopamina. Noutro estudo, procurou-se examinar as conexões entre alterações no sistema serotoninérgico e o padrão do apego numa amostra de adultos adotados. Os resultados desse estudo indicaram que o polimorfismo funcional no gene transportador de serotonina ${ }^{1}$ (i.e., 5-HTTLPR), na variante alélica curta, parece exercer influência sobre o funcionamento da amígdala, que se trata de uma estrutura relacionada com a reatividade e regulação emocional. Constatou-se uma associação significativa entre este traço genético particular e o padrão de apego desorganizado/ desorientado (Caspers et al., 2009).

Gênero. Em particular no domínio sócio-emocional, têm sido identificadas diferenças de gênero em adotados. Wiik et al. (2011), num estudo realizado nos Estados Unidos da América, verificaram que rapazes adotados de instituições da Europa do Leste e da Ásia evidenciavam mais problemas externalizados e internalizados, bem como déficit de atenção e hiperatividade, em comparação com as raparigas adotadas. A estes resultados acrescentam-se outros que mencionam que as crianças adotadas do sexo

\footnotetext{
${ }^{1}$ Serotonina ou 5-hidroxitriptamina (5-HT) é um neurotransmissor associado a transtornos do humor e alterações comportamentais.
}

masculino que experienciaram cuidados institucionais tendem a apresentar uma maior falta de seletividade nas relações (Roy, Rutter, \& Pickles, 2004). Noutro estudo observou-se que os adotados adultos do sexo masculino estavam em maior risco de apresentar uma perturbação do humor, quando comparados com adultos adotados do sexo feminino e adultos não adotados de ambos os sexos (Tieman, Van der Ende, \& Verhulst, 2005).

Experiências Pré-Natais. Simmel (2007), com base numa amostra de 293 crianças que foram adotadas no seu país de origem (i.e., Estados Unidos da América), apurou que $57 \%$ das crianças tinham estado expostas, no período pré-natal, a drogas ou álcool (57\%) e a nicotina (27\%). Relativamente ao consumo de drogas, no estudo Canadense de Nulman et al. (1994), no qual crianças adotadas expostas a cocaína durante a gravidez foram comparadas com crianças também adotadas mas não expostas, constatou-se que as primeiras tinham nascido, em média, cerca de três semanas antes (37 versus 40 semanas) e pesavam menos ao nascimento. No entanto, três anos depois do nascimento, os dois grupos não diferiam em termos de peso e de comprimento, mas o perímetro cefálico das crianças expostas era significativamente menor. Os grupos também não diferiam em termos de QI, mas as crianças expostas apresentavam uma performance significativamente inferior na linguagem. Mais recentemente, Crea, Shenyang, Barth e Brooks (2008) examinaram os efeitos da exposição a substâncias ilícitas nos comportamentos externalizados, 14 anos após a adoção. Os autores analisaram as trajetórias de desenvolvimento de 275 crianças e concluíram que a exposição pré-natal predizia a ocorrência de mais problemas de conduta.

Aronson, Kyllerman, Sabel, Sandin e Alegard (1985) estudaram 21 crianças adotadas no seu país de origem, a Suécia, e cujas mães biológicas consumiram álcool durante a gravidez. Aos nove anos de idade, o QI das crianças expostas era significativamente inferior ao QI das crianças não expostas. Landgren, Svensson, Strömland e Grönlund (2010) encontraram uma associação entre o alcoolismo materno durante a gravidez e déficits cognitivos e motores, bem como a presença de problemas como autismo e déficit de atenção/hiperatividade, cinco anos depois da adoção. O consumo excessivo de álcool durante a gestação pode resultar na Síndrome do Alcoolismo Fetal (SAF), cujo diagnóstico inclui três características principais (Streissguth \& Connor, 2001): (a) um padrão específico de anomalias craniofaciais; (b) déficits pré e pós-natais no comprimento e/ou peso; e (c) danos cerebrais ou disfunção. A incidência de SAF foi identificada em 16\% de crianças adotadas nos Estados Unidos da América e provenientes de países da Europa do Leste (D. E. Johnson et al., 1996). Neste estudo, apesar de outras crianças não apresentarem um diagnóstico, as suas histórias de vida, traços físicos e padrão de crescimento sugeriram a presença de SAF.

Quando se procura avaliar os resultados desenvolvimentais em crianças adotadas, e que anteriormente foram expostas a experiências desfavoráveis, devem ser 
Baptista, J. S., Soares, I. C. \& Henriques, M. R. (2013). Recuperação Desenvolvimental Após a Adoção: Características da Criança e da Família Adotiva.

considerados os fatores de risco ambiental vivenciados no período pré-natal, para uma compreensão aprofundada da (in)adaptação posterior destas crianças. Todavia, os estudos mencionados foram elaborados com base em relatos da família adotiva, verificando-se uma ausência de investigações, cujos dados tenham sido obtidos de forma direta e junto da família biológica.

Experiências de Maus-Tratos e História de Cuidados. Antes da adoção, muitas das crianças foram vítimas de maus-tratos, negligência e/ou abuso sexual por parte dos pais biológicos ou da família extensa, podendo estas circunstâncias terem-se constituído como o motivo que conduziu à retirada da criança do lar biológico. Todavia, constatou-se uma notória falta de estudos sobre o impacto dos maus-tratos no desenvolvimento da criança adotada. Este fato deve-se à escassez de informação sobre a história que antecedeu a adoção. Simmel (2007) identificou, numa amostra já mencionada neste artigo, percentagens elevadas de crianças que tinham sido vítimas de negligência $(42 \%)$, abuso sexual (13\%) e maus-tratos físicos $(22 \%)$. Constatou ainda que aquelas vítimas de negligência e de abuso sexual estavam em maior risco de apresentar problemas de internalização e externalização, o que é consistente com outros estudos (Van IJzendoorn \& Juffer, 2006). Por sua vez, Kertes, Gunnar, Madsen e Long (2008) identificaram uma associação significativa entre a história de abuso e negligência da criança adotada (medida com base nos relatos retrospectivos dos pais adotivos norte americanos) e déficits ao nível do crescimento físico no momento da adoção. A amostra era constituída por crianças provenientes de instituições da Europa do Leste, Ásia e América do Sul.

Aqueles resultados são indicadores de que a história de cuidados que antecede a adoção poderá exercer uma influência considerável em diferentes domínios do desenvolvimento da criança adotada. A este respeito, importa realçar os resultados de estudos, mais e menos recentes, que analisaram a associação entre os cuidados prestados em contexto institucional e o desenvolvimento da criança. Tizard, Cooperman, Joseph e Tizard (1972) exploraram a relação entre a qualidade dos cuidados prestados em instituições Inglesas e o desenvolvimento da linguagem, tendo verificado que as crianças que estavam institucionalizadas nas estruturas de melhor qualidade (i.e., maior estabilidade em termos de staff e cuidadores experientes) apresentavam resultados médios no desenvolvimento da linguagem acima dos valores de referência. Variáveis como (a) o comprimento médio das frases dos cuidadores, (b) a complexidade das mesmas, e (c) o envolvimento em atividades de estimulação estavam também associadas a um melhor desempenho na linguagem. Smyke et al. (2007), por sua vez, identificaram uma associação positiva entre a sensibilidade do cuidador em instituições Romenas e o desenvolvimento cognitivo da criança. As crianças expostas a cuidados de maior qualidade apresentavam menos problemas externalizados.
Estes resultados apontam para a relevância da qualidade dos cuidados prestados em contexto institucional. Todavia, constatou-se uma preocupante ausência de estudos dedicados à análise da influência exercida pelas características estruturais e relacionais das instituições no desenvolvimento da criança, meses e anos após a adoção. Esta circunstância deve-se ao reduzido número de estudos longitudinais, dedicados à monitorização da trajetória de desenvolvimento da criança, desde a sua integração na instituição até anos após a adoção. A acumulação de evidência empírica acerca do impacto do acolhimento institucional na infância é ainda hoje, essencialmente, indireta, baseada nos relatos da família adotiva e em variáveis como o tempo de institucionalização e idade na adoção.

Alguns investigadores (e.g., Roy et al., 2000) hipotetizaram que as crianças que antes da adoção experienciaram cuidados em famílias de acolhimento apresentam resultados mais favoráveis do que as crianças que estiveram institucionalizadas, na medida em que o acolhimento familiar tende a caracterizar-se pela possibilidade de ocorrência de um maior número de interações entre a criança e um cuidador primário, bem como possibilita que a criança usufrua de cuidados mais consistentes e individualizados. Em um estudo levado a cabo com crianças adotadas por famílias norte americanas, Miller, Chan, Comfort e Tirella (2005) constataram que as crianças que anteriormente tinham estado institucionalizadas na Guatemala apresentavam valores inferiores de comprimento, peso e perímetro cefálico no momento da adoção, em comparação com crianças que tinham estado em famílias de acolhimento. Por sua vez, Loman, Wiik, Frenn, Pollack e Gunnar (2009) compararam dois grupos de crianças: (a) o primeiro grupo era composto por crianças adotadas que estiveram institucionalizadas durante mais de $75 \%$ das suas vidas e que foram integradas em famílias adotivas norte americanas quando tinham 12 ou mais meses de idade; (b) o segundo grupo era composto por crianças adotadas com oito ou menos meses de idade, que não estiveram institucionalizadas durante mais de dois meses, sendo que $65 \%$ experienciaram apenas cuidados em famílias de acolhimento antes da adoção. As crianças que tinham estado em instituições eram provenientes da Europa do Leste, Ásia, América do Sul e África. Os autores constataram que, no momento da adoção, as crianças que experienciaram cuidados institucionais apresentavam valores inferiores de peso e comprimento, comparativamente com as crianças adotadas antes dos oito meses de idade. Entre os oito e os 11 anos de idade, as crianças foram reavaliadas: para o peso e para o desenvolvimento cognitivo e da linguagem, as crianças que tinham estado em instituições continuavam a revelar valores mais baixos do que as crianças que tinham estado em famílias de acolhimento. Quanto às dificuldades de atenção e hiperatividade, Wiik et al. (2011) verificaram que as crianças que tinham estado em acolhimento institucional antes da adoção apresentavam níveis mais elevados de problemas, do que as crianças que previamente tinham estado em famílias de acolhimento. 
Os resultados identificados nos estudos anteriores têm sido consistentemente apoiados por outras investigações (e.g., Wilson, Weaver, Cradock, \& Kuebli, 2008).

Tempo de Institucionalização e Idade na Adoção. $\mathrm{O}$ enfoque colocado em estudos sobre a adoção internacional, realizados com crianças provenientes de países onde práticas como a institucionalização são comuns, conduziu ao uso recorrente da variável tempo de institucionalização (associada à idade na adoção e à quantidade de fatores de risco) como uma medida representativa da adversidade precoce ao nível dos cuidados (Gunnar \& Kertes, 2005). Em termos do crescimento físico, do desenvolvimento cognitivo e aprendizagem é ampla a evidência acerca das relações existentes entre o tempo de institucionalização e déficits nestes domínios, meses e anos após a adoção (e.g., Van IJzendoorn \& Juffer, 2006; Van IJzendoorn et al., 2005). Este padrão de resultados tem sido identificado noutros domínios, como no desenvolvimento da linguagem (Glennen \& Maters, 2002) e motor (Londen, Juffer, \& Van IJzendoorn, 2007). Além destes, num estudo piloto com 14 adolescentes adotados por famílias do Reino Unido, e que tinham estado institucionalizados na Romênia (Mehta et al., 2009), verificou-se que o volume da amígdala esquerda estava correlacionado com o tempo de institucionalização: os adolescentes que evidenciavam um menor volume eram os que tinham permanecido durante mais tempo institucionalizados.

Relativamente aos problemas de comportamento, verificou-se que crianças adotadas tendiam a evidenciar mais problemas de internalização, quanto mais tempo tinham estado em acolhimento institucional (Wiik et al., 2011). Alguma literatura (e.g., Stevens et al., 2008) tem igualmente sugerido que crianças com história de institucionalização mais prolongada estão em risco particular de apresentarem dificuldades de atenção e hiperatividade. Outros demonstraram que crianças adotadas depois da infância apresentavam mais problemas de externalização (Merz \& McCall, 2010). Contudo, um estudo anterior contraria este resultado (Rutter et al., 2001), no qual se verificou que, aos seis anos de idade, crianças Romenas adotadas tardiamente por famílias do Reino Unido não evidenciavam mais problemas, quando comparadas com crianças adotadas antes dos seis meses de idade. Com efeito, a evidência empírica não é conclusiva a este respeito, o que denota a importância da realização de mais estudos, permitindo compreender a complexidade do tema.

Quanto à segurança do apego, numa meta-análise concluiu-se que as crianças que foram adotadas antes dos 12 meses de idade não se diferenciavam dos pares da comunidade (Van den Dries, Juffer, Van IJzendoorn, \& Bakermans-Kranenburg, 2009). Numa publicação recente foi divulgado que a integração numa família de acolhimento antes dos 24 meses de idade parece funcionar como um fator protetor para a formação de relações de apego seguras (Smyke, Zeanah, Fox, Nelson, \& Guthrie, 2010). Este resultado, bem como os anteriores, poderão ser indicadores da importância da integração da criança numa família durante um período sensível para a formação de uma relação de apego e adaptação posterior, bem como da relevância da brevidade da exposição ao contexto institucional.

Apesar da consistência dos resultados anteriores, outros autores (e.g., Castle et al., 1999) argumentam ainda que a relação entre a idade no momento da adoção e o desenvolvimento posterior persiste nos casos em que a criança experienciou antes da adoção cuidados de menor qualidade, sendo nestes casos que a adoção mais tardia prediz piores resultados desenvolvimentais. Se assim é, consideramos que não só a idade no momento da adoção se reveste de importância. A análise dos efeitos da idade da criança no momento em que foi institucionalizada é igualmente relevante. Num estudo, constatou-se que as crianças institucionalizadas precocemente, na Coreia, devido a abandono eram as que evidenciavam mais problemas de externalização quando comparadas com crianças que foram institucionalizadas mais tarde (Lee, Seol, Sung, \& Miller, 2010). R. Johnson, Browne e Hamilton-Giachritsis (2006) concluíram que crianças institucionalizadas antes dos 24 meses de idade apresentavam mais comportamentos perturbados de apego de tipo indiscriminado do que crianças que foram institucionalizadas mais tarde. Assim sendo, quanto mais precocemente a criança for exposta a experiências de privação, mais traumática essa experiência parece ser; e quanto mais tempo a criança estiver exposta a tais condições, menos completa será a recuperação física e psicossocial posterior.

Estatuto Desenvolvimental Prévio. Qual o impacto do estatuto desenvolvimental da criança anterior à adoção nos ganhos posteriores? Pormerleau et al. (2005) verificaram que, ao longo dos primeiros seis meses de integração na família adotiva, as crianças que apresentavam ganhos mais acentuados no domínio cognitivo e motor eram as que evidenciavam um desempenho inicial inferior, ou seja, no momento de chegada à família adotiva. Também Judge (2003) identificou resultados que vão nesta direção, apurando que o número de déficits no desenvolvimento no momento da adoção era um preditor dos ganhos efetuados entre a chegada à família e o sexto mês de integração. Destacamos ainda o estudo de Croft et al. (2007), no qual se constatou que a presença de linguagem na adoção estava associada ao funcionamento cognitivo posterior. No momento em que foram adotadas, a maioria das crianças apresentava déficits ao nível do desenvolvimento da linguagem. Porém, aquelas que tinham pelo menos 18 meses de idade e eram capazes de se envolver em comportamentos verbais de imitação foram as que apresentaram resultados mais positivos em termos de funcionamento cognitivo aos 11 anos de idade. Na medida em que a competência linguística estava associada à qualidade do contexto institucional, os autores sugeriram que as variações no desenvolvimento da linguagem seriam uma consequência da qualidade da experiência institucional. 
Baptista, J. S., Soares, I. C. \& Henriques, M. R. (2013). Recuperação Desenvolvimental Após a Adoção: Características da Criança e da Família Adotiva.

Os resultados dos estudos anteriores indicam que a mudança para um contexto familiar pode proporcionar oportunidades para a recuperação em domínios distintos do funcionamento da criança. Alertam também para o fato de que as experiências institucionais podem exercer uma influência considerável no grau de recuperação posterior. Características institucionais que podem afetar os resultados do desenvolvimento da criança pós-institucionalizada devem ser, assim, consideradas, como, por exemplo, a nutrição inadequada, a ausência de momentos privilegiados de estimulação e a inexistência de relações de apego seletivas.

\section{Contributos do Contexto Familiar Adotivo}

O número de estudos que examinaram os componentes da qualidade do ambiente familiar envolvidos nos ganhos desenvolvimentais após a adoção é consideravelmente menor do que o número de estudos dedicados aos resultados desenvolvimentais da criança. Todavia, que variáveis demográficas e que competências dos pais adotivos parecem contribuir para a heterogeneidade desenvolvimental identificada na criança adotada? No que diz respeito à relação entre a idade dos pais e o desenvolvimento, os investigadores não têm identificado associações significativas, o mesmo acontecendo com o nível socioeconômico e a escolaridade (e.g., Wilson et al., 2008). Contudo, a investigação com adotantes tem-se caracterizado pela falta de heterogeneidade entre participantes, nas variáveis referidas.

Sensibilidade Parental. De acordo com Ainsworth, Bell e Stayton (1974), as diferenças individuais evidenciadas ao nível da qualidade da organização do apego estão associadas a histórias distintas de interação da criança com a sua figura de apego: um apego seguro está relacionado com cuidados mais sensíveis; um apego inseguro-evitante está associado a cuidados rejeitantes e não responsivos; e um apego inseguro-ambivalente relaciona-se com cuidados insensíveis e inconsistentemente responsivos. A sensibilidade parental pode ser definida como a competência do adulto para perceber os sinais da criança e responder de forma adequada e pronta (Ainsworth et al., 1974). Além disto, a investigação tem realçado que o comportamento responsivo é essencial para o desenvolvimento das competências linguísticas, cognitivas, sociais e emocionais (Landry, Smith, Miller-Loncar, \& Swank, 1997).

Num estudo longitudinal com famílias adotivas holandesas, constatou-se que a sensibilidade materna aos seis meses de idade da criança é um preditor do desenvolvimento cognitivo aos sete anos de idade (Stams, Juffer, Rispens, \& Hoksbergen, 2000). Quanto ao apego, aquela equipe de investigadores constatou que a sensibilidade materna não estava associada significativamente à (des) organização do apego, ao contrário da hipótese formulada pelos autores (Londen et al., 2007). Curiosamente, noutro estudo observou-se que os déficits cognitivos e a presença de problemas de comportamento em crianças adotadas tendiam a fazer emergir nos pais adotivos comportamentos menos positivos. Estes comportamentos, por sua vez, sofreram uma diminuição ao longo do tempo, a par com a evolução positiva das competências da criança (Croft et al., 2001). Estes autores vieram realçar a influência das características da criança na qualidade da relação criança/ pais adotivos, colocando um enfoque na natureza interativa e dinâmica dos mecanismos envolvidos na mudança.

Modelos Internos de Funcionamento. Estudos têm salientado a relevância dos modelos internos de funcionamento do adulto (i.e., representações mentais acerca do self, das figuras de apego e das relações entre os dois) na formação de uma relação segura de apego. Representações (in)seguras estão associadas a (in)sensibilidade parental e a uma relação de apego (in)segura entre a criança e o adulto (Van IJzendoorn, 1995). Num estudo conduzido em Portugal, Veríssimo e Salvaterra (2006) identificaram uma associação positiva entre a segurança do apego da criança e o modelo interno de funcionamento da mãe adotiva: as crianças cujas mães adotivas evidenciaram a utilização de um script de base segura tendiam a apresentar valores de segurança.

Aquela variável foi alvo de análise num estudo Espanhol (Palacios, Román, Moreno, \& Léon, 2009), no qual foram observadas diferenças significativas entre os pais adotivos e os pais da comunidade ao nível do modelo interno de funcionamento, apresentando os pais da comunidade resultados mais favoráveis. Os autores sugeriram que este resultado pode ser um reflexo da influência das experiências adversas vividas anteriormente pela criança no modelo interno de funcionamento dos pais adotivos. Estudos com famílias de acolhimento têm revelado o impacto das características da criança na qualidade da relação criança/pais, evidenciando que no período inicial de formação de uma relação de apego entre a criança e a família, os comportamentos de resistência e de evitamento da criança contribuem para o emergir de comportamentos de rejeição no adulto, mesmo em adultos seguros/autônomos (Stovall-McClough \& Dozier, 2004). Os resultados dos estudos mencionados sugerem que dificuldades da criança adotada podem ser um desafio, mesmo para adultos com capacidades parentais consideradas adequadas. Serviços de pós-adoção são, desta forma, fundamentais.

\section{Conclusão}

Os resultados dos estudos mencionados no presente artigo vão ao encontro da proposta de Rutter (2005), segundo a qual a investigação sobre o impacto da adoção deverá estar assente na combinação de três fatores - experiências pré-natais, de cuidados anteriores e posteriores à adoção, e a constituição genética. A heterogeneidade no grau e tipo de déficit é uma característica dos resultados desenvolvimentais de crianças adotadas que estiveram privadas de cuidados parentais adequados. Em algumas se constata, por exemplo, que a institucionalização prolon- 
gada pode levar a déficits físicos e cognitivos acentuados e/ou a problemas de comportamento. Outras crianças não evidenciam dificuldades. Recursos ambientais, genéticos e temperamentais podem estar envolvidos nas diferenças observadas. Neste sentido, é fundamental o estudo da interação entre as características da criança e as características dos contextos nos quais aquela se movimenta, tanto antes como depois da adoção. Um olhar sobre as interações entre crianças resilientes e diferentes facetas dos contextos poderá levar ao entendimento sobre como melhorar a vida institucional e familiar.

Todavia, consideramos que as razões subjacentes à heterogeneidade observada no desenvolvimento não são ainda plenamente compreendidas, não se esgotando nas variáveis referidas neste artigo. Há necessidade de mais investigações dedicadas à análise da influência de experiências específicas de cuidados no desenvolvimento da criança adotada, em culturas distintas. Déficits nos cuidados, em particular na prestação de cuidados responsivos e estáveis em contexto institucional, permanecem como questões de interesse. Aquela análise revela-se premente para a identificação de fatores de risco e proteção capazes de esclarecer a possibilidade dos seus efeitos variarem conforme a idade no momento de integração na família adotiva, o tempo de institucionalização e a vulnerabilidade genética. A identificação dos fatores explicativos daquela heterogeneidade, que moderam os caminhos entre o risco e a desordem, representa uma etapa fundamental para a compreensão científica do fenômeno, bem como para a melhoria das práticas clínicas junto destas crianças e suas famílias.

Apesar da descrição dos resultados do desenvolvimento após a adoção ser necessária, consideramos que esta perspectiva desligada de um entendimento dos processos que ocorrem no seio da família, não permite alcançar uma compreensão aprofundada das dinâmicas envolvidas no fenômeno da adoção. Estudos futuros devem obter informações detalhadas sobre características diversas dos pais adotivos (e.g., motivações subjacentes à adoção, práticas educativas parentais), de outras variáveis indicadoras da qualidade da relação (e.g., atenção partilhada, comunicação afetiva), bem como informações acerca da família biológica, nomeadamente acerca de fatores genéticos e ambientais (e.g., experiências de maus-tratos). Uma análise aprofundada de resultados específicos de domínios mais amplos de funcionamento da criança (e.g., déficits de atenção, enquadrados nos problemas de externalização), em subamostras de adotados, poderá ser uma estratégia promissora, bem como a análise do período de surgimento de diferentes tipos de problemas.

A investigação sobre a adoção deve-se pautar pela ocorrência de outras movimentações, como de estudos transversais para longitudinais. Devido ao seu caráter menos dispendioso, os investigadores têm recorrido a desenhos transversais. Contudo, tais desenhos são limitados na procura de um entendimento acerca das trajetórias de desenvolvimento da criança adotada. Entendemos como essencial a realização de estudos longitudinais, com início no momento de chegada da criança à instituição, possibilitando a análise das experiências anteriores à institucionalização e da própria institucionalização. Esta elaboração poderá ser ainda um veículo privilegiado para testar se a recuperação no crescimento físico, no desenvolvimento cognitivo e sócio-emocional, e se a construção de relações seguras de apego está limitada pelo nível de privação vivenciado precocemente.

Além disto, a investigação sobre a adoção tem estado centrada na comparação entre crianças adotadas e seus pares da comunidade. Todavia, esta estratégia poderá não ser a mais adequada, devido ao fato de condições desiguais estarem a ser alvo de comparação. Consideramos mais pertinente a comparação entre crianças adotadas e as que permaneceram institucionalizadas, pois ambas estiveram expostas a experiências adversas e a histórias de separação parental, mas as segundas não foram integradas num contexto familiar adotivo.

A investigação com famílias adotivas reveste-se de inúmeros desafios, dada a diversidade de influências que podem ocorrer na família, bem como devido ao fato de cada criança levar consigo para o novo lar histórias distintas de cuidados. Sugerimos a possibilidade de se constituir uma base de dados conjunta, entre vários países, de forma a permitir à comunidade científica explorar os efeitos da variável cultural, bem como desenvolver estudos focados em características particulares de diferentes tipos de famílias e de crianças.

\section{Referências}

Ainsworth, M., Bell, S., \& Stayton, D. (1974). Infant-mother attachment and social development: Socialization as a product of reciprocal responsiveness to signals. In M. P. Richards (Ed.), The integration of a child into a social world (pp. 99-135). London: Cambridge University Press.

American Psychiatric Association. (2000). Diagnostic and statistical manual of mental disorders ( $4^{\text {th }}$ Rev. Ed.). Washington, DC: Author.

Aronson, M., Kyllerman, M., Sabel, K., Sandin, B., \& Alegard, R. (1985). Children of alcoholic mothers: Developmental, percetual and behavioral characteristics as compared to matched groups. Ata Psychiatrica Scandinavia, 74, 27-35.

Bronfenbrenner, U. (1993). The ecology of cognitive development: Research models and fugitive findings. In R. Wonziak \& K. Fischer (Eds.), Development in context: Acting and thinking in specific environments (pp. 3-44). Hillsdale, NJ: Erlbaum.

Caspers, K., Paradiso, S., Yucuis, R., Troutman, B., Arndt, S., \& Philibert, R. (2009). Association between the serotonin transporter promoter polymorphism (5-HTTLPR) and adult unresolved attachment. Developmental Psychology, 45(1), 64-76.

Castle, J., Groothues, C., Bredenkamp, D., Beckett, C., O'Connor, T. G., Rutter, M., \& The ERA Study Team. (1999). Effects of quality of early institutional care on cognitive attainment. American Orthopsychiatric Association, 69(4), 424-437. 
Baptista, J. S., Soares, I. C. \& Henriques, M. R. (2013). Recuperação Desenvolvimental Após a Adoção: Características da Criança e da Família Adotiva.

Cohen, N., Lojkasek, M., Zadeh, Z. Y., Pugliese, M., \& Kiefer, H. (2008). Children adopted from China: A prospective study of their growth and development. Journal of Child Psychology and Psychiatry, 49(4), 458-468.

Crea, T., Shenyang, G., Barth, R., \& Brooks, D. (2008). Behavioral outcomes for substance-exposed adopted children: Fourteen years postadoption. American Journal of Orthopsychiatry, 78(1), 11-19.

Croft, C., Beckett, C., Rutter, M., Castle, J., Colvert, E., Groothues, C., ...Sonuga-Barke, E. (2007). Early adolescent outcomes of institutionally-deprived and non-deprived adoptees II: Language as a protective fator and a vulnerable outcome. Journal of Child Psychology and Psychiatry, 48(1), 31-44.

Croft, C., O’Connor, T. G., Keaveney, L., Groothues, C., Rutter, M., \& The English and Romanian Adoption Study Team. (2001). Longitudinal change in parenting associated with developmental delay and catch-up. Journal of Child Psychology and Psychiatry, 42, 649-659.

Glennen, S., \& Masters, M. G. (2002). Typical and atypical language development in infants and toddlers adopted from Eastern Europe. American Journal of Speech and Language Therapy, 11, 417-433.

Gunnar, M., \& Kertes, D. (2005). Prenatal and postnatal risks to neurobiological development in internationally adopted children. In D. Brodzinsky \& J. Palacios (Eds.), Psychological issues in adoption (pp. 47-66). London: Praeger.

Johnson, D. E. (2000). Medical and developmental sequelae of early childhood institutionalization in Eastern European adoptees. In C. Nelson (Ed.), The effects of early adversity on neurobehavioral development. Minnesota Symposia on Child Psychology (Vol. 31, pp. 113-162). Mahwah, NJ: Erlbaum.

Johnson, D. E., Albers, L, Iverson, S., Mathers, M., Dole, K., Georgieff, M., ...Miller, L. (1996). Health status of Eastern European orphans referred for adoption. Pediatric Research, 39(4), 134A

Johnson, R., Browne K. D., \& Hamilton-Giachritsis, C. E. (2006). Young children in institutional care at risk of harm. Trauma Violence and Abuse, 7(1), 34-160.

Joint United Nations Programme on HIV, United Nations Children's Fund, \& United States Agency for International Development. (2004). Children on the brink 2004: A joint report of new orphan estimates and a framework for action. Geneva, Switzerland: Joint United Nations Programme on HIV.

Judge, S. (2003). Developmental recovery and deficit in children adopted from Eastern European orphanages. Child Psychiatry and Human Development, 34(1), 49-62.

Juffer, F., \& Rosenboom, L. (1997). Infant-mother attachment of internationally adopted children in the Netherlands. International Journal of Behavioral Development, 20(1), 93-107.

Kertes, D., Gunnar, M., Madsen, N., \& Long, J. (2008). Early deprivation and home basal cortisol levels: A study of internationally adopted children. Developmental and Psychopathology, 20(2), 473-491.

Landgren, M., Svensson, L., Strömland, K., \& Grönlund, M. A. (2010). Prenatal alcohol exposure and neurodevelopmental disorders in children adopted from Eastern Europe. Pediatrics, 125(5), 1178-1185.

Landry, S., Smith, K., Miller-Loncar, C., \& Swank, P. (1997). Responsiveness and initiative: Two aspects of social competence. Infant Behavior and Development, 20(2), 263-266.
Lee, R., Seol, K., Sung, M., \& Miller, M. (2010). The behavioral development of Korean children in institutional care and international adoptive families. Developmental Psychology, 46(2), 468-478.

Loman, M., Wiik, K. L., Frenn, K., Pollack, S., \& Gunnar, M. (2009). Postinstitutionalized children's development: Growth, cognitive, and language outcomes. Journal of Development \& Behavioral Pediatrics, 30(5), 426-434.

Londen, M., Juffer, F., \& Van IJzendoorn, M. (2007). Attachment, cognitive, and motor development in adopted children: Short-term outcomes after international adoption. Journal of Pediatric Psychology, 32(10), 1249-1258.

Marcovitch, S., Cesaroni, L., Roberts, W., \& Swanson, C. (1995). Romanian adoption: Parents' dreams, nightmares, and realities. Child Welfare: Journal of Policy, Practice, and Program, 74(5), 993-1017.

Mehta, M., Golembo, N., Nosarti, C., Colvert, E., Mota, A., Williams, S., ...Sonuga-Barke, E. (2009). Amygdala, hippocampal and corpus callosum size following severe early institutional deprivation: The English and Romanian Adoptees Study Pilot. Journal of Child Psychology and Psychiatry, 50(8), 943-951.

Merz, E., \& Mcall, R. (2010). Parent ratings of executive functioning in children adopted from psychosocially depriving institutions. Journal of Child Psychology and Psychiatry, 52(5), 537-546

Miller, L., Chan, W., Comfort, K., \& Tirella, L. (2005). Health of children adopted from Guatemala: Comparison of orphanage and foster care. Pediatrics, 115(6), 710-717.

Nulman, I., Rovet, J., Altmann, D., Bradley, C., Einarson, T., \& Koren, G. (1994). Neurodevelopment of adopted children exposed in utero to cocaine. Canadian Medical Association Journal, 151, 1591-1597.

O'Connor, T. G., \& Zeanah, C. (2003). Introduction to the special issue: Current perspetives on assessment and treatment of attachment disorders. Attachment and Human Development, 5(3), 221-222.

Palacios, J., Román, M., Moreno, C., \& Léon, E. (2009). Family context for emotional recovery in internationally adopted children. International Social Work, 52(5), 609-620.

Pormerleau, A., Malcuit, G., Chicoine, J., Séguin, R., Belhumeur, C., Germain, P., ...Jéliu, G. (2005). Health status, cognitive and motor development of young children adopted from China, East Asia, and Russia across the first 6 months after adoption. International Journal of Behavioral Development, 29(5), 445-457.

Roy, P., Rutter, M., \& Pickles, A. (2000). Institutional care: Risk from family background or pattern of rearing? Journal of Child Psychology and Psychiatry, 41, 139-149.

Roy, P., Rutter, M., \& Pickles, A. (2004). Institutional care: Associations between overactivity and lack of selectivity in social relationships. Journal of Child Psychology and Psychiatry, 45(4), 866-873.

Rutter, M. (2005). Adverse preadoption experiences and psychological outcomes. In D. Brodzinsky \& J. Palacios (Eds.), Psychological issues in adoption (pp. 67-92). London: Praeger.

Rutter, M., Kreppner, J., O'Connor, T. G., \& The ERA Study Team. (2001). Specificity and heterogeneity in children's responses to profound privation. British Journal of Psychiatry Special Issue, 179, 97-103.

Simmel, C. (2007). Risk and protective factors contributing to the longitudinal psychosocial well-being of adopted foster 
children. Journal of Emotional and Behavioral Disorders, $15,237-249$.

Smyke, A., Koga, S., Johnson, D., Fox, N., Marshall, P. J., Nelson, C., ...The BEIP Core Group. (2007). The caregiving context in institution-reared and family-reared infants and toddlers in Romania. Journal of Child Psychology and Psychiatry, 48, 210-218.

Smyke, A., Zeanah, C., Fox, N., Nelson, C., \& Guthrie, D. (2010). Placement in foster care enhances quality of attachment among young institutionalized children. Child Development, 81(1), 212-223.

Stams, G. J., Juffer, F., Rispens, J., \& Hoksbergen, R. (2000). The development and adjustment of 7-year old children adopted in infancy. Journal of Child Psychology \& Psychiatry, 8, 1025-1037.

Stevens, S., Kumsta, R., Kreppner, J., Brookes, K., Rutter, M., \& Sonuga-Barke, E. (2009). Dopamine transporter gene polymorphism moderates the effects of severe deprivation on ADHD symptoms: Developmental continuities in gene-environment interplay. American Journal of Medical Genetics Part B: Neuropsychiatric Genetics, 150B(6), 753-761.

Stevens, S., Sonuga-Barke, E., Kreppner, J., Beckett, C., Castle, J., Colvert, E., ...Rutter, M. (2008). Inattention/overactivity following early severe institutional deprivation: Presentation and associations in early adolescence. Journal of Abnormal Child Psychology, 36, 385-398.

Stovall-McClough, K. C., \& Dozier, M. (2004). Forming attachments in foster care: Infant attachment behaviors during the first 2 months of placement. Development and Psychopathology, 16, 253-271.

Streissguth, A. P., \& Conner, P. D. (2001). Fetal alcohol syndrome and other effects of prenatal alcohol: Developmental cognitive neuroscience implications. In C. Nelson \& M. Luciana (Eds.), Handbook of developmental cognitive neuroscience (pp. 505-518). Cambridge, MA: Massachusetts Institute of Technology Press.

Tan, T., \& Yang, Y. (2005). Language development of Chinese adoptees 18-35 months old. Early Childhood Research Quarterly, 20(1), 57-68.

Tieman, W., Van der Ende, J., \& Verhulst, F. C. (2005). Psychiatric disorders in young adult intercountry adoptees: An epidemiological study. American Journal of Psychiatry, 162(3), 592-598.

Tizard, B., Cooperman, O., Joseph, A., \& Tizard, J. (1972). Environmental effects on language development. A study of young children in long-stay nurseries. Child Development, 43, 337-358.

Van den Dries, L., Juffer, F., Van IJzendoorn, M., \& Bakermans-Kranenburg, M. (2009). Fostering Security? A meta-analysis of attachment in adopted children. Children and Youth Services Review, 31, 410-421.

Van IJzendoorn, M. (1995). Adult attachment representations, parental responsiveness, and infant attachment - A meta-analysis on the predictive-validity of the Adult Attachment Interview. Psychological Bulletin, 117, 387-403.

Van IJzendoorn, M., \& Juffer, F. (2006). The Emanuel Miller Memorial Lecture 2006: Adoption as intervention. Meta-analytic evidence for massive catch-up and plasticity in physical, socioemotional, and cognitive development. Journal of Child Psychology and Psychiatry, 47(12), 1228-1245.

Van Ijzendoorn, M., Juffer, F., \& Klein-Poelhuis, C. (2005). Adoption and cognitive development: A meta-analytic comparison of adopted and nonadopted children's IQ and school performance. Psychological Bulletin, 131, 301-316.
Veríssimo, M., \& Salvaterra, F. (2006). Maternal secure-base scripts and children's attachment security in an adopted sample. Attachment and Human Development, 8(3), 261-273.

Wiik, K. L., Loman, M., Van Ryzin, M. J., Armstrong, J. M., Essex, M. J., Pollak, S., \& Gunnar, M. (2011). Behavioral and emotional symptoms of post-institutionalized children in middle childhood. The Journal of Child Psychology and Psychiatry, 52(1), 56-63.

Wilson, S., Weaver, T., Cradock, M., \& Kuebli, J. (2008). A preliminary study of the cognitive and motor skills acquisition of young international adoptees. Children and Youth Services Review, 30, 585-596.

Zeanah, C., Smyke, A., Koga, S., Carlson, E., \& The Beip Core Group. (2005). Attachment in institutionalized and community children in Romania. Child Development, 76, 1015-1028.
Recebido: 04/08/2011

$1^{a}$ revisão: 13/01/2012

$2^{a}$ revisão: $27 / 04 / 2012$

Aceite final: 30/04/2012 